\title{
Proyectos de inversión: Un enfoque diferente de análisis
}

\begin{abstract}
RESUMEN
El presente artículo brinda un enfoque conceptual distinto sobre los proyectos de inversión, considerando la flexibilidad de análisis en distintas realidades de intervención e incorporando dimensiones de estudio necesarios para lograr una sostenibilidad de las inversiones en los entornos actuales.
\end{abstract}

Palabras clave: Proyecto de inversión, análisis de enfoque.

Investment Projects:

A DIFFERENT APPROACH OF ANALYSIS

\section{ABSTRACT}

This article provides a different conceptual approach on investment projects, considering the flexibility of analysis in different realities of intervention, and incorporating dimensions of study necessary to achieve sustainability of investments in today's environment.

Key words: Investment projects, investment, social approach, sustainability, profitability.

\section{INTRODUCCIÓN}

Toda organización sea del ámbito privado o público se encuentra en constante mejora de sus servicios, ya sea en términos de calidad o cobertura, para ello requiere realizar inversiones, las que deben generar ciertos niveles de rentabilidad; es aquí donde los proyectos de inversión se convierten en el medio para la adecuada gestión de inversiones, permitiendo estimar con anticipación los resultados de dichas intervenciones.

En algunas ocasiones por las condiciones, del mercado y factores que influyen en los negocios, las inversiones no logran los objetivos deseados, situación que se presenta por aspectos sociales, ambientales o propios de la identificación del proyecto.

Debido a la complejidad de las intervenciones empresariales y los constantes cambios de los escenarios donde se desarrollan, se hace necesario adecuar los conceptos de proyectos de inversión, que permitan un análisis integral desde distintas perspectivas y puedan abordar de una mejor manera las inversiones.

Por tanto, el objetivo del artículo es proporcionar una visión conceptual diferente de los proyectos de inversión que permita una flexibilidad de adecuación a distintas realidades de análisis.

\section{PROYECTO DE INVERSIÓN}

El proyecto de inversión desde una perspectiva general, se entiende como una intervención en un medio para dar solución a una problemática existente y lograr un cambio deseado. Dicho problema se puede percibir como una limitación o un exceso de un bien y/o servicio.

\section{Se caracteriza porque:}

- Es una intervención delimitada en el tiempo.

- Se integra como una unidad de servicio en un horizonte de tiempo.

- Interviene en un medio social: población y organizaciones.

1 Ingeniero Industrial UNMSM, Profesor en la Facultad de Ingeniería Industrial, Departamento Académico de Producción y Gestión Industrial de la UNMSM.

Email: cieandia@hotmail.com 
- Interviene en un área geográfica definida donde tendrá influencia el proyecto

Ejemplos:

Proyecto de diversificación de productos en una empresa industrial

- Su intervención debe tener un tiempo de ejecución: 8 meses.

- Unidad de servicio: Línea de producción y comercialización del producto

- El medio social se refiere a la población de la zona de intervención: población del departamento de Lima.

- El área geográfica es el ámbito donde tendría influencia el proyecto: departamento de Lima.

Proyecto de mejora de pistas y veredas

- Su intervención debe tener un tiempo de ejecución: 5 meses.

- Servicio de transporte y tránsito durante 10 años luego de terminado la etapa de ejecución.

- El medio social se refiere a la población de la zona: 500 familias.

- El área geográfica es el ámbito donde tendría influencia el proyecto: Asentamiento Humano " $X$ ".

Proyecto de Ampliación de servicios educativos

- Su intervención debe tener un tiempo de ejecución: 6 meses.

- Servicio educativo durante 10 años luego de terminado la etapa de ejecución.

- El medio social se refiere a la población de la zona: 200 familias.

Figura 1. Proyecto de inversión desde la perspectiva de proceso

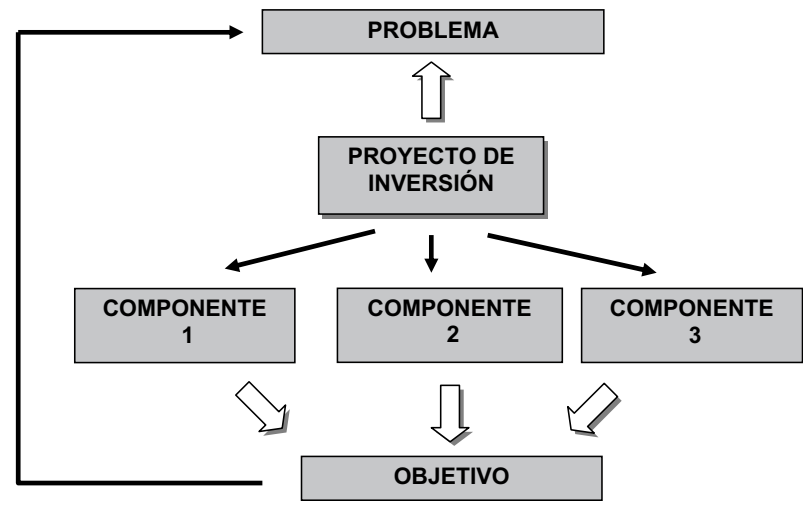

Fuente: Elaboración propia.
- El área geográfica es el ámbito donde tendría influencia el proyecto: Distrito "A".

La definición anterior permite una flexibilidad conceptual en los distintos "medios" de intervención, es decir si el medio de intervención es el ambiente, nos referimos a los proyectos ambientales; si el medio es social, nos referimos a proyectos sociales.

Desde la perspectiva de proceso el proyecto se define como un conjunto de actividades interdependientes agrupadas en componentes orientadas al logro de un objetivo en un tiempo determinado (Figura N. ${ }^{\circ}$ 1). Es decir, para intervenir un problema identificado el proyecto tiene que incorporar varios componentes (obra, equipamiento, capacitación, etc.) que en conjunto lograran cumplir el objetivo y a su vez resolver el problema.

Desde la perspectiva de estudio (documento), el proyecto de inversión es aquel donde se plasma con claridad y detalle lo que se desea lograr; además, permite justificar la intervención desde distintos puntos de vista para dar solución a una problemática, por lo que se debe explicar las siguientes interrogantes:

\begin{tabular}{|l|l|}
\hline \multicolumn{1}{|c|}{ Pregunta } & \multicolumn{1}{|c|}{ Tema del Proyecto } \\
\hline ¿Qué se desea hacer? & $\begin{array}{l}\text { Naturaleza del } \\
\text { proyecto }\end{array}$ \\
\hline $\begin{array}{l}\text { ¿Por qué es necesario } \\
\text { realizar el proyecto? }\end{array}$ & $\begin{array}{l}\text { Justificación del } \\
\text { proyecto }\end{array}$ \\
\hline $\begin{array}{l}\text { ¿Para qué se va realizar } \\
\text { el proyecto? }\end{array}$ & Objetivo del proyecto \\
\hline $\begin{array}{l}\text { ¿Cuáles son los cambios } \\
\text { a realizar? }\end{array}$ & Metas \\
\hline $\begin{array}{l}\text { ¿A quién va dirigido el } \\
\text { proyecto? }\end{array}$ & $\begin{array}{l}\text { Beneficiarios directos } \\
\text { e indirectos }\end{array}$ \\
\hline $\begin{array}{l}\text { ¿Dónde se va a imple- } \\
\text { mentar el proyecto? }\end{array}$ & Ámbito geográfico \\
\hline $\begin{array}{l}\text { ¿Cómo se va a interve- } \\
\text { nir? }\end{array}$ & Actividades \\
\hline $\begin{array}{l}\text { ¿Cuánto de recursos se } \\
\text { requiere? }\end{array}$ & Inversión \\
\hline $\begin{array}{l}\text { ¿Cuáles son los benefi- } \\
\text { cios a obtener? }\end{array}$ & Rentabilidad \\
\hline
\end{tabular}

Los Proyectos de Inversión desde la perspectiva organizacional son intervenciones sobre los procesos existentes (conjunto de actividades que se complementan para generar un servicio) cuyo ob- 
jetivo es mejorar el servicio de la organización. Por lo tanto, es una intervención temporal.

\section{Ejemplo:}

- Proyecto de inversión para mejorar los servicios administrativos de una institución del Estado

- Proyecto de Inversión para la automatización de procesos en una empresa industrial

Proyecto de Inversión desde una perspectiva de generación de nuevas iniciativas empresariales es un modelo en el cual, en base a un conjunto de información y antecedentes, se puede estimar las ventajas y desventajas que se derivan de asignar ciertos recursos para la producción de un bien o la realización de un servicio, que permiten satisfacer necesidades en forma adecuada, creativa y eficiente.

Por tanto, los proyectos empresariales pueden solucionar problemas u oportunidades de negocio, ambas tienen en común satisfacer necesidades insatisfechas. En muchos casos las oportunidades son resultados de analizar de una manera diferente los problemas.

\section{DIMENSIONES DE ANÁLISIS DE LOS PRO- YECTOS: LA DIMENSIÓN SOCIAL, ENFOQUE INDISPENSABLE EN LOS PROYECTOS}

Para que los proyectos logren un desarrollo sostenible en sus distintas etapas, tienen que ser analizados desde tres dimensiones distintas, la primera

Figura 2. Proyecto de inversión desde la perspectiva organizacional

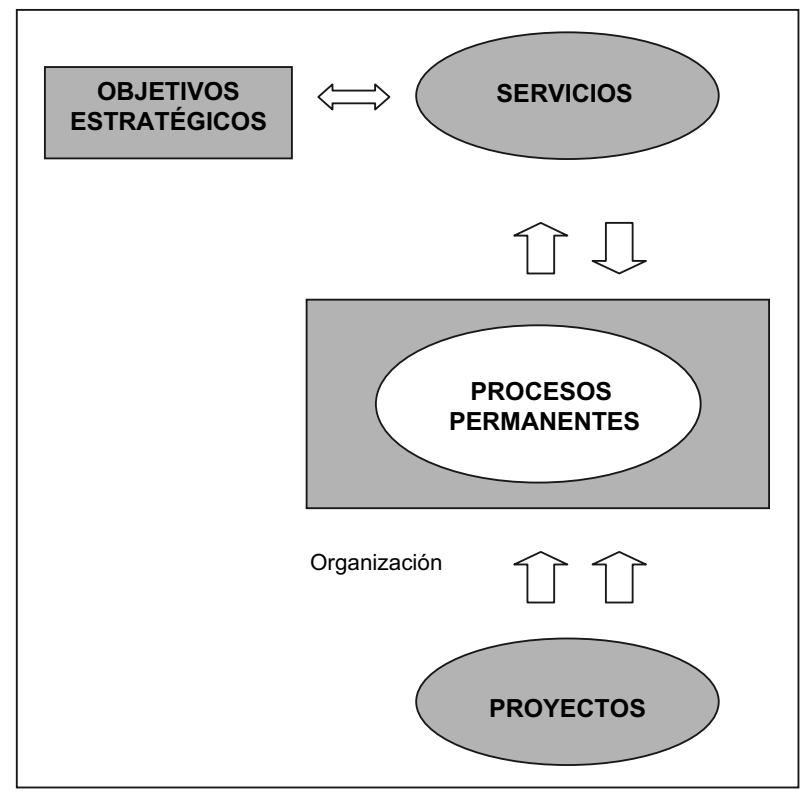

Fuente: Elaboración propia.
Figura 3: Proyecto de inversión desde la perspectiva empresarial.

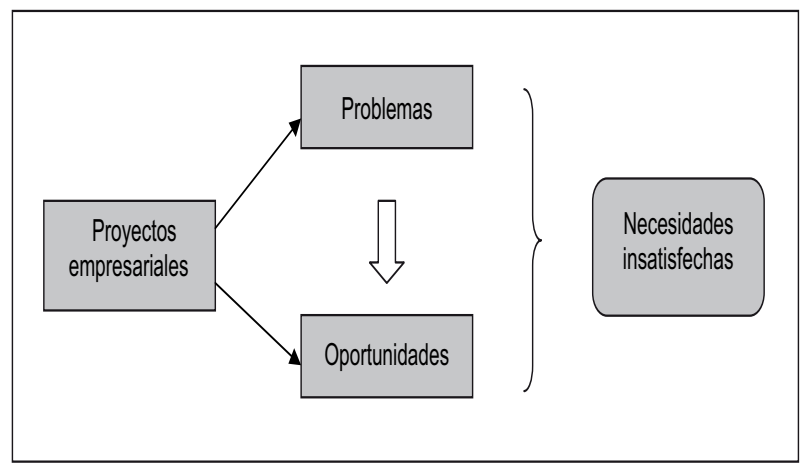

Fuente: Elaboración propia.

es la dimensión intrínseca, en la cual se analizan los impactos directos e indirectos propios de la naturaleza del proyecto; por ejemplo, para un proyecto de carreteras tiene como impacto el ahorro de tiempo, reducción de uso de combustibles, etc., para un proyecto de salud los impactos propios del proyecto se relacionan a ese tema.

La segunda dimensión de análisis es la ambiental, es decir, todo proyecto puede generar impactos positivos o negativos al medio ambiente donde se desarrolla, los que deben ser identificados para incluirlos dentro de la evaluación.

La tercera dimensión de análisis es la social, es decir todo proyecto se desarrolla en un medio donde existen poblaciones, organizaciones, que debido a sus costumbres, historias, creencias, políticas, formas de organización, etc., serán beneficiadas o perjudicadas por cambios en dichas características. Por lo que, es importante conocer dichos grupos de interés involucrados, incluir en el análisis sus percepciones sobre el tema; el no hacerlo puede generar rechazo al proyecto.

El análisis de la dimensión social se presenta en forma independiente a la dimensión, ambiental porque es importante identificar si va a existir algún tipo de rechazo por parte de grupos de interés debido a los cambios generados en sus condiciones sociales (costumbres, hábitos, etc.) que no tenga relación con los impactos ambientales que causan daño a la población.

Una vez identificados todos los impactos por cada una de las dimensiones se valoran y luego se incorporan en la evaluación del proyecto, con efectos tanto en la inversión como en los costos de implementación. 
El esquema de análisis propuesto busca resaltar la importancia del análisis del medio social debido a la multiplicación de los conflictos sociales que se presentan, así como evitar la duplicidad de información y estudios que se realizan; por ejemplo, en muchos casos, se considera los estudios socioeconómicos dentro de la dimensión ambiental, siendo dicha información analizada en la dimensión intrínseca.

El análisis de la dimensión social se presenta en forma independiente a la dimensión ambiental porque es importante identificar si va existir algún tipo de rechazo por parte de grupos de interés debido a los cambios generados en sus condiciones sociales que no tenga relación con los impactos ambientales que causan daño a la población.

\section{CONCLUSIONES}

1. La gestión de las inversiones constituye un aspectos fundamental en las organizaciones por lo que su análisis está en constante evolución conceptual y de procedimientos metodológicos.

2. Es necesario incorporar una visión conceptual flexible en la definición de los proyectos de inversión, para que se pueda abordar de una manera adecuada las inversiones considerando los nuevos escenarios donde se desarrollan.
3. Para que los proyectos sean sostenibles desde los distintos aspectos, es decir, compatibles con el medio ambiente, sin conflictos sociales y con una rentabilidad económica, es importante incluir en su análisis las tres dimensiones: intrínseca, ambiental y social.

\section{REFERENCIAS BIBLIOGRÁFICAS}

1. ANDÍA VALENCIA, W. (2010). Gerencia de Proyectos Sociales y de Inversión Social. 1. ${ }^{\text {ra }}$ ed. El Saber Editores. Perú.

2. ANDÍA VAlENCIA, W. (2009). Proyectos de Inversión. 2. ${ }^{a}$ ed. El Saber Editores. Perú.

3. ANDÍA VALENCIA, W. (2009). Manual de gestión ambiental. 1. ${ }^{\text {ra }}$ ed. El Saber Editores. Perú.

4. CASTRO, R. y Mokate K. (1994). Evaluación económica de proyectos de inversión, Facultad de Economía, Universidad de los Andes, Colombia.

5. COHEN, E. y FRANCO, R. (1992). Evaluación de proyectos sociales. Editorial Siglo XXI, México.

6. FONTAINE, E. (1997). Evaluación social de proyectos. $11 .^{\text {ra }}$ ed. Chile.

Figura 4: Dimensiones de análisis de los Proyecto de Inversión

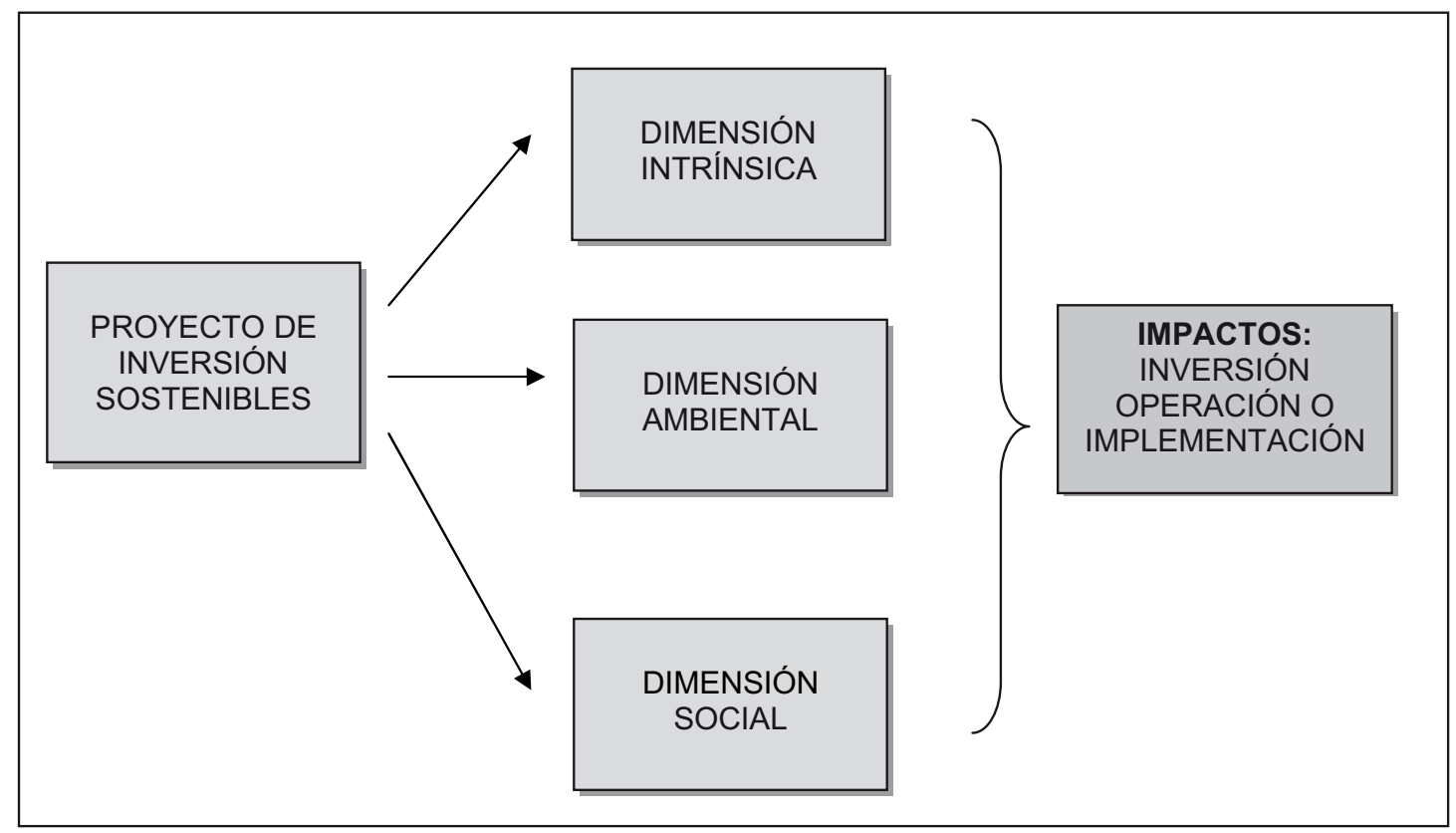

Fuente: Elaboración propia. 